\title{
Étude sur modèle des mesures à prendre pour améliorer le dégravement d'une prise d'eau existante
}

\author{
(Prise de la Soulce sur la Durance)
}

\section{Model studies of the necessary steps for improving the desilting of an existing intake}

\author{
PAR MII. LABAYE WT FACCONNTER

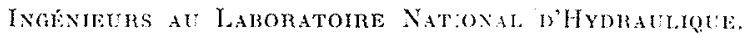

English synopsis, p. 578 .

\section{INTRODUCTION}

Liobjel de cette note est de souligner quelques idées générales sur le problème de l'engravement des prises d'eau, dégagées au cours d'étude sur modèle réduit effectué au Laboratoire National d'Hydraulique, et de montrer dans quelle mesure elles ont pu etre appliquées au cas d'une prise d'eau existante : la prise de la Saulce sur la Durance. Cette dernière étude a été entreprise en étroite collaboration avec le Service de la Production Hydratulque de l'Exploitation Electricité de France.

\section{I. - La rivière et les ouvrages}

Le bassin versant de la Durance à la prise de la Saulce est d'environ $4.000 \mathrm{~km}$. Son régime est caractéristique des rivières alpines du versant méditerrancen : avec un module légìrement supérieur à $100 \mathrm{~m}^{*} / \mathrm{s}$, il présente de hautes caux de printemps, d'avril à juillet, allant jusquà $500 \mathrm{~m} " / \mathrm{s}$; les plus fortes crues, correspondant a des précipitations courtes et violentes, montent à $600 \mathrm{~m}^{3} / \mathrm{s}$ et exceptionnellement jusqu'à $1.000 \mathrm{~m} / \mathrm{s}$. Leur durée est relativement courte : un ou deux jours par an.

Le lit mineur divague dans un lit majeur très large (plus de $500 \mathrm{~m}$ par endroit) sur un fond de granulométric relativement étenduc : $d_{s, 1}=40 \mathrm{~nm} ; d_{90}=90 \mathrm{~mm}$. Le débit de commen-

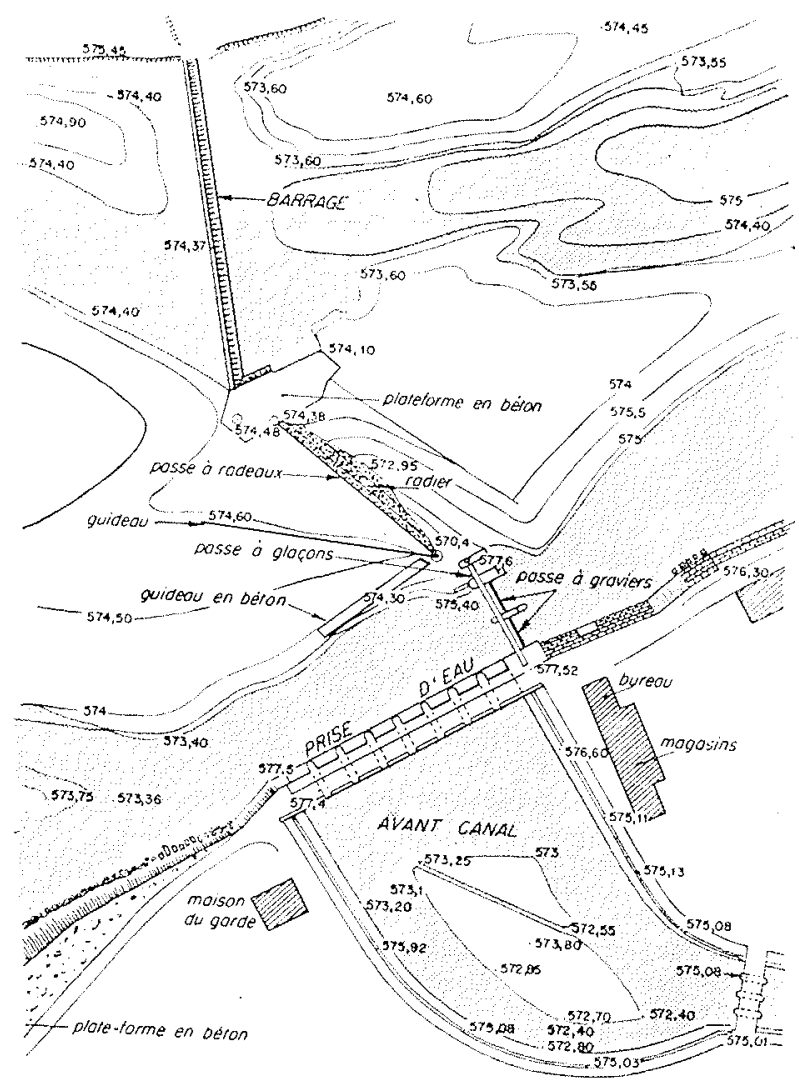

Fic. 1. Ourrages de prise de la saulce dans lour atal actucl. 
cement d'entrainement a ćté évalué à environ $130 \mathrm{~m}^{2} / \mathrm{s}$.

On voit la disposition actuelle des ouvrages sur la figure 1.

Le barrage a en plan la forme d'un $V$ dont les branches, dirigées vers l'aval et faisant entre elles un angle de $110^{\circ}$, ont respectivement pour longueur $115 \mathrm{~m}$ el $54 \mathrm{~m}$. Il avait à l'origine $2,5 \mathrm{~m}$ environ de hauteur, mais se trouve actuellement entièrement engravé, si bien qu'il n'y a pratiquement plus de retenue.

La prise elle-mème comporte 8 pertuis débouchant dans un avant-canal qui communique avec le canal proprement dit par un ouvrage de garde (ef. fig. 1 et 3 ). On voit sur la figure 3 l'état d'engravement de l'avant-canal. Il comporte d'ailleurs un dessableur à trémies pyramidales qui s'est trouvé complètement obstrué au bout de quelques jours de fonctionnement. Une partic des matériaux est, par des manouvres appropriées des vannes de prise, entrainée dans te canal où l'on en effectue le dragage. On extrait ainsi jusqu'à $10.000 \mathrm{~m}^{3}$ par an de gravier.

Le débit dérivé, de $40 \mathrm{~m}^{3} / \mathrm{s}$ à l'origine, a été successivement porté à 62 , puis à $82 \mathrm{~m}^{3} / \mathrm{s}$. On envisage de l'augmenter de 25 at $30 \mathrm{~m} / \mathrm{s}$, mais on a aetuellement beaucoup de difficultés pour obtenir le débit de $82 \mathrm{~m}^{3} / \mathrm{s}$.

Le dispositif de chasse est constitué par deux vannes de $8 \mathrm{~m}$ de largeur, placées côte à côte immédiatement à l'aval de la prise et perpendieutatrement a celle-ci (ct. fig. 1 et 3). Elles sont separes du harage proprement dit par une petite passe à glacons. Leur seuil semble avoir été placé sensiblement à $1 \mathrm{~m}$ en dessous du fond naturel de la rivière.

Le lit mineur naturel à l'amont du barrage n'avait aucune position stable au point qu'il s'est trouvé en 1947 rejeté completement sur la rive gauche à la suite d'une crue moyeme. Cette

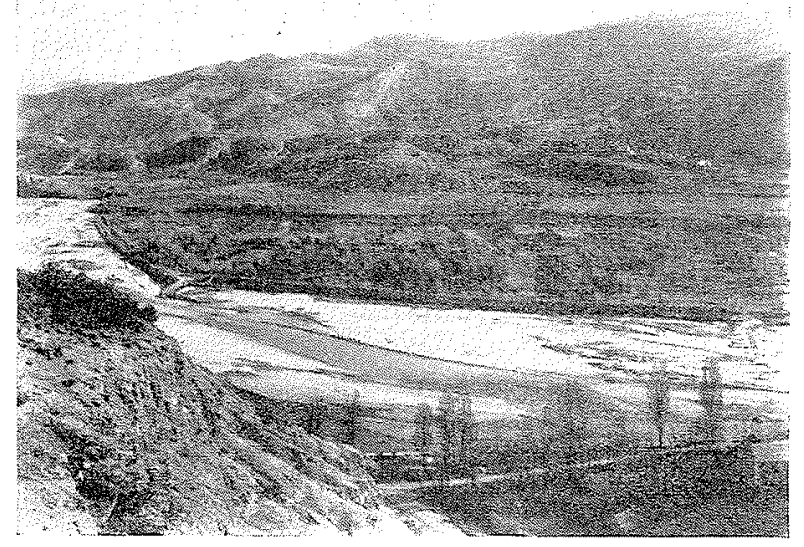

Fia. 2. - Aspect du lit de la Durance a l'amont de la prise de la Saulce.

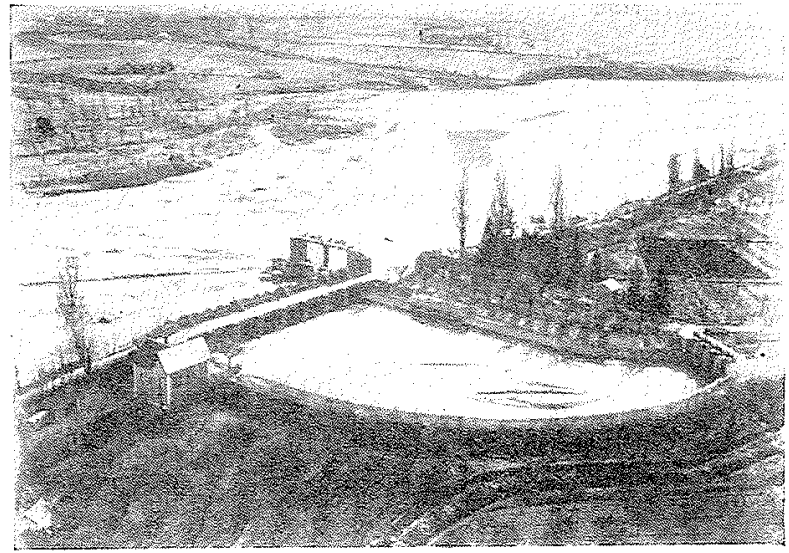

Fic. 3. - Vue de la prise dans son état actuel. Aspect du lit à l'aval du barrage.

même instabilité caractérise le lit mineur à l'abal du barrage (cf. fig. 2 et 3 ).

\section{II. - Généralités}

De toute évidence, nous nous trouvons en présence d'une rivière roulant sur ses propres alluvions. Il semble mème, compte tenu de la dimension des plus gros matériaux, que le lit de la Durance doive présenter assez peu le phénomène de pavage, c'est-à-dire que, dès que le débit dépasse sensiblement celui pour lequel le matériau commence à cheminer sur le fond, la plupart des éléments du lit se mettent en mouvement.

$\mathrm{Si}$, pour une telle rivicre, on fait l'analyse des différents paramètres qui en régissent les caractéristiques : profils en long et en travers du lit en particulicr, un certain nombre d'hypothèses se présentent à l'esprit, qu'il serait intéressant de vérifier, peut-être par une étude statistique.

La pente générale de la rivière, que l'ingénieur, sinon le géographe, peut considérer comme étant en équilibre, n'est-elle pas réglée par les apports solides annuels globaux, dus en majorité aux crues moyennes, comple tenu de la granulométrie des matériaux el de l'ilendue du bassin versant?

La forme du lit en travers ne serait-elle pas sous l'influence plus directe de la facon dont ces apports solides se repartissent en fonction des débits liquides? Une riviere, pour laquelle le charriage commence à un débit relativement faible, pour croitre progressivement lorsque le débit augmente, aurait un profil en travers plus aplati qu'une autre riviere qui, lout en transportant annuellement la même quantilé de matériaux, verrait son charriage commencer pour un débit liquide plus important, mais croître rapidement avec celui-ci. 
Ce sont là des hypothèses très génćrales; il est bien évident quil y aura lieu de faire intervenir les dimensions des matériaux et l'étendue de leur granulométrie qui dépend elle-mème vraisemblablement du régime de la rivière et en particulier de la soudaineté et de la violence des crues, ainsi que de létendue du bassin versant. Si elles ne sont pas en contradiction avec les observations que nous arons pu faire sur les différents essais en cours d'étude au Laboratoire, elles ne peuvent ividemment être confirmées ou infirmées que par une étude suffisamment vaste des rivieres naturelles. Les mesures que te Service des Etudes et Recherches Hydrauliques entreprend actuellement dans la region des Alpes seront, à cet égard, extrêmement intéressantes.

De toute facon, l'implantation d'un barrage de prise au fil de l'eau modifie sensiblement les conditions locales et, si l'on ne connaît pas encore les lois que l'on pourrait déduire des hypotheses ci-dessus ou d'autres analogues, on sait que la riviere modifiera son profil en long ou son profil en travers ou les deux, de facon a s'adapter à ces nouvelles conditions.

Ce qui nous manque encore, ee sont des ordres de grandeur suffisamment précis pour que l'on sache si l'on a intérèt à modifier artificiellement de facon profonde des profils en travers, en conservant sensiblement la mème pente au lit, ou inversement a admettre une modification notable de la pente locale au prix d'un calibrage plus facile des sections amont et aval. Ne serait-on pas limité d'ailleurs dans les transformations a faire subir a la section transversale, par des conditions de stabilité de formes, indépendantes des phénomènes ei-dessus?
Dans le cas de la prise étudice avec un débit dérivé qui, à l'heure actuelle, est relativement près du débil de commencement d'entrainement, le problème des chasses continues est assez délicat. En effet, pour évacuer les apports solides a l'aval du barrage avec un débit de chasse restreint, il faut an préalable les avoir rassemblés à proximité de cette chasse. Ce rassemblement peut se faire en utilisant au maximum les possibilités naturelles offertes par la riviere, par exemple : coude qui devra être dans le sens convenable aussi bien pour le lit mineur que pour le lit majeur. Mais ceci nest efficace que si les conditions d'écoulement jusqu'au voisinage des chasses sont telles que le charriage soit assuré correctement.

Ces quelques idées, dont on aurait dù d'ailleurs étudier les modalités d'application sur modèle dans le cas d'une prise nouvelle, ont dû s'accommoder, pour létude de la prise de la Saulee, des ouvrages existant, qu'il convenait de conserver au maximum. Il faut remarquer d'ailleurs que, lorsque cette prise a ité construite aux environs de 1910 , le débit dérivó ćtait de $40 \mathrm{~m} 3 / \mathrm{s}$ seulement, alors qu'il a dé doublé depuis.

Nous allons maintenant essayer de préciser les modalités dapplieation de ces idées générales, en prenant comme exemple la prise d'eau de la Saulce. Nous développerons d'abord le probleme de l'évacuation continue des matériaux à l'aval des ouvrages qui conditionnait la róussite du projet, puis nous décrirons les mestures prises à l'amont ef nous terminerons par lélude des dispositifs de chasse proprement dits.

\section{EVACUATION DES MATERIAUX A L'AVAL DU BARRAGE}

\section{I. - Position du problème}

Pour des conditions à l'amont et des ourrages donnés, l'efficacite de tout dispositif de chasse dépend, pour un débit donné de la rivière, du débit qu'il peut évacuer et de la différence de niveau existant entre l'amont et l'aval.

Le problème du dégravement est particulièrement difficile, dans le cas présent, pour les débits compris entre $Q_{0}$ : débil de commencement de charriage, et $2 \mathrm{Q}_{0}$.

C'est done dans cet intervalle critique, et plus particulierement pour les débits très peu supérieurs à $Q_{i}$, qu'il faut, d'une part réaliser le maximum de dénivellation, mais d'autre part assurer la slabilite de la cote aval choisie comme la plus favorable. Or cette dernière condition va a l'encontre de la tendance naturelle à l'exhaussement constatée à laval de toute installation de prise dans une riviere à charriage notable.

L'exemple étudié de la prise de la saulee a l'intérêt de poser le problème avec beaucoup d'acuité : il s'agissait de maintenir une évacuation continue du débit solide $G$ de l'amont, à travers un lit majeur trois fois plus large, avee des débits liquides diminués du débit dérivé et avec une pente à peu près imposée, sensiblement la même que la pente initiale.

\section{II. -- Principe de résolution}

C'est done pour le débit $\mathrm{Q}_{0}-\mathrm{Q}_{n}\left(\mathrm{Q}_{n}=\right.$ débit dérivé), e'est-à-dire lorsque les matériaux com- 
menceront à être évacués par les chasses, que l'on doit maintenir la cole aval de facon à oblenir une dénivellation suffisante et stable.

Il s'agit de choisir, dans ce but, le profil en long du fond de la rivicre à l'aval du harrage en abaissant d'une part la cote immédiatement a laval des ouvrages de chasse et, d'autre part, en réalisant un lit mineur à pente maximum rejoignant les conditions naturelles suffisamment à l'aval.

En troisieme lien, la pente étant ainsi fixée, ce lit mineur artificiel doit etre calibré afin de satisfaire à une loi de variation de son rayon hydraulique $R$ en fonction de la hauteur d'eatu, qui corresponde au mieux aux deux conditions de charriage suivantes :

1" Pour le débit $Q_{0}-Q_{r}$ assurer les condilions d'équilibre, c'est-ádire l'entrainement continu des materiaux;
2" Pour les débils liquides plus importants, disposer d'une capacité de charriage égale ou tris légèrement supérieure à la loi $\mathrm{G}$ $\left(\mathrm{Q}-\mathrm{Q}_{n}\right)$ du charriage de la Durance. It vaut mieux en effet risquer un abaissement du fond, quilte a le limiter ensuite a l'aide d'un pavage artificiel.

\section{III. - Application à la prise de la Saulce (cf. fig. 4)}

On a d'abord admis qu'il suffisait de rejoindre d'une manière continue les conditions naturelles existant au droit du resserrement du pont de Curbans, c'est-à-dire sur une longueur d'environ 1.300 mètres.

On a alors ramene la cote du fond immédiatement à l'aval des ouvrages de ehasse à sa valeur ancienne, soit à un peu plus de $2 \mathrm{~m}$ au-des-

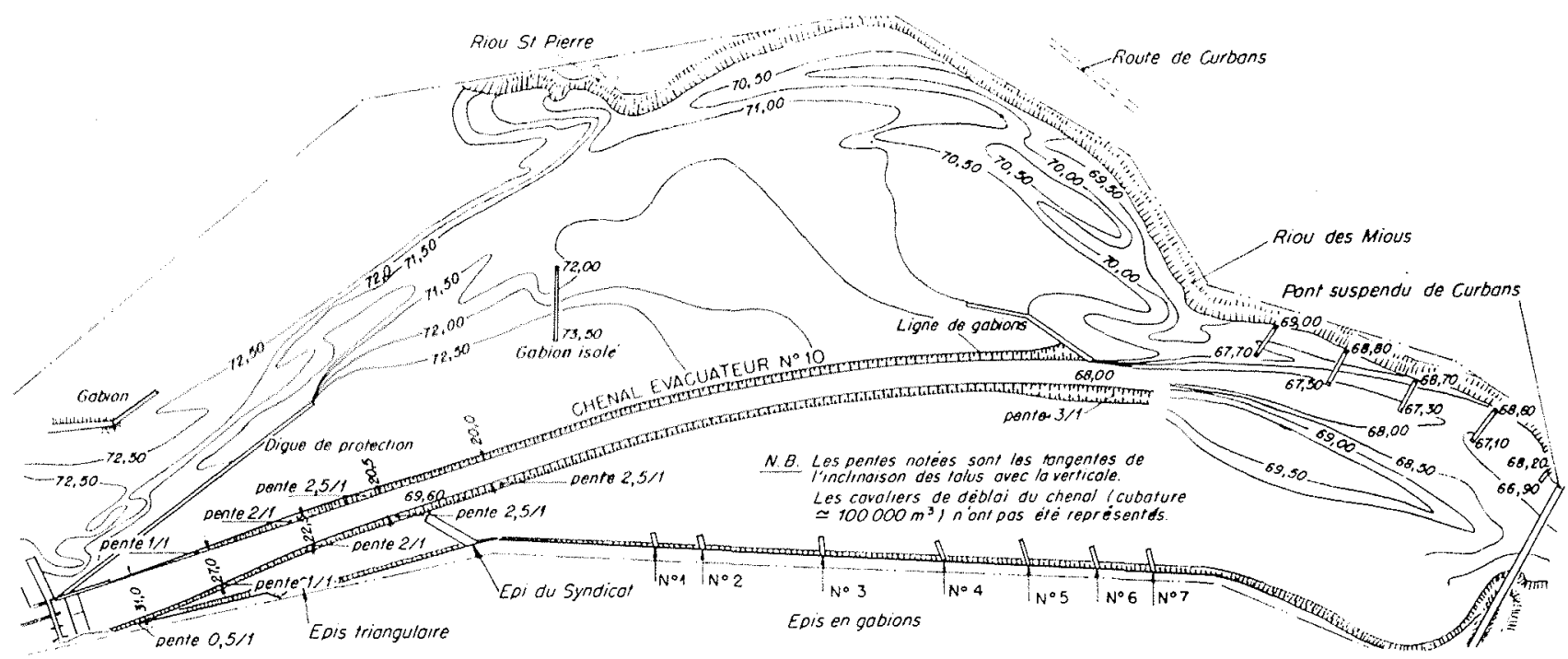

Fig. 4.

Essat de régularisation du lit de la Durance a l'aval des ourages

sous de la cote actuelle. Le tracé en plan, adopté pour des raisons qui seront expliquées plus loin, donne au profil on long du fond une pente d'environ $3 \%$.

On ne pouvait ainsi disposer que d'une pente sensiblement égale a celle qui devait exister initialement. C'est pourquoi on a constaté qu'un calibrage du lit à l'aide d'épis plongeants s'avérait insuffisamment stable et efficace. Il a done fallu, pour obtenir des résultats satisfaisants, étudier une solution à l'aide d'un chenal évacuateur. On a alors déterminé une section du chenal telle que le plafond reste stable pour un dé- bit d'environ $50 \mathrm{~m} \% / \mathrm{s}\left(Q_{0}-Q_{i j}\right)$, ce qui a conduit à la nécessité d'une cunctte centrale de $20 \mathrm{~m}$ de largeur.

Enfin, on a complété cette cunette par ún élargissement progressif limitant au maximum les afrouillements dans le chenal pour les débits supérieurs (ef. fig. 5).

La solution proposée, bien (que satisfaisant au but recherché, présentera cependant l'inconvénient grave d'une difficile réalisation à cause des fortes vitesses dans le chenal au cours des crues.

Il se forme cependant pendant les tres forles 


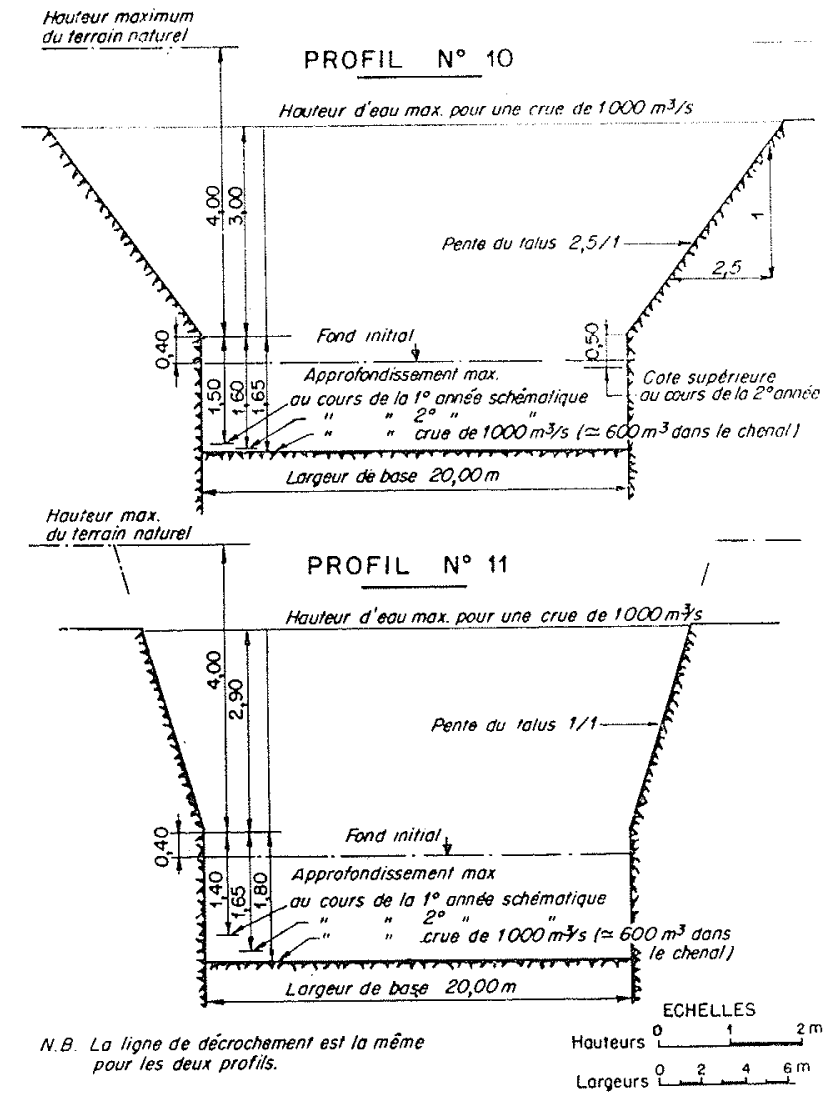

Fic. 5. - Prise de la Saulce. Chenal évacuateur. crues un second lit alimenté par déversement, à travers la zone du lit majeur aval situce à gauche du chenal évecuateur. On a donc dû étudier les dispositions it prendre afin de slabiliser ce lit intermittent dans tequel de charriage n'aura dailleurs lieu en moyenne fu'm jour par an) dans une position poll laqueile il n'y ait pas it crandre dattaque du cavaier gatuche du chenal crachateur, ceci mène dans le cas de certaines conditions execptionnelles (travaux exécul's sur le chenal ou les ouvages) (ef. fig. 4 !.

Signalons enfin une autre particularite intéressante de l'étude. Afin de diminuer d'environ $350 \mathrm{~m}$ la longueur effective du chenal, on a été conduil à réaliser un tracé en courbe de ce chenal, avec sortie tangentielle à la berge rive ganche, de telle sorte que celle-ci se trouve attaruée dis' les débits moyens : l'effet de courbure favorisant le dépót des matériaux sur la rive droite, il suffit dans ces conditions de protéger la berge rive gauche ainsi menacée par quelques dérenses en gabions. Avec cette disposition, le lit se maintient jusqu'au resserrement du pont de Curbuns d'une manière continue el stable quel que soit le débit. On assure ainsi un passage progressif d'un calibrage rigide aux conditions naturelles (1).

(1) Des cssais complementaires ont montre depuis qu'on pouvait datleurs se contenter de defendre la vive convexe du chenal par des épis.

\section{CORRECTION DU LIT A L'AMONT DU BARRAGE}

On sait qu'il y a intérèt à disposer une prise d'eau en rivière après un coude du lit mincur, tangentiellement à la rive concave. Par suite des courants transversaux créés par ce coude, la zone de charriage se trouve alors localisée sur une bande plus ou moins large du iit, entre la rive convexe et la souille qui se forme le lons de la rive concave. Cependant, ajoutons qu'il ne suffit pas toujours de choisir, ou d'obtenir, par une correction, cette courbure du lit mineur; il est souvent utile de calibrer artificiellement le lit afin de limiter l'étendue du remous solide créé par le barrage, même si l'on ne peut pas créer ou fixer un coude.

Comme on l'a signalé ci-dessus, ces conditions ne sont pas réalisées dans le cas de la prise de la Saulce. Avant la construction en 1948 de l'épi guideau rive gauche, le lit mineur pouvait divaguer d'une manicre queleonque suivant le regime de la Durance; depuis, il semble stabilisé sur la rive droite et ne présente plus de coude. On a donc élé amené à fixer et calibrer artificiellement ce lit à l'aide d'épis, selon une courbe relativement stable, quels que soient le débit et les mo-

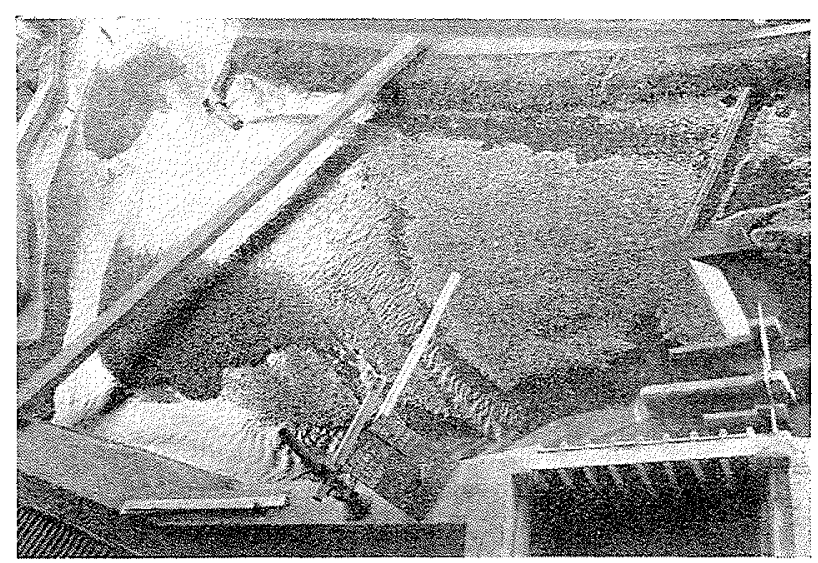

Fic. 6. - Aspect du lit á l'amont du barrage sans régularisation.

Le bras rive droite seul charrie des matériaux. 


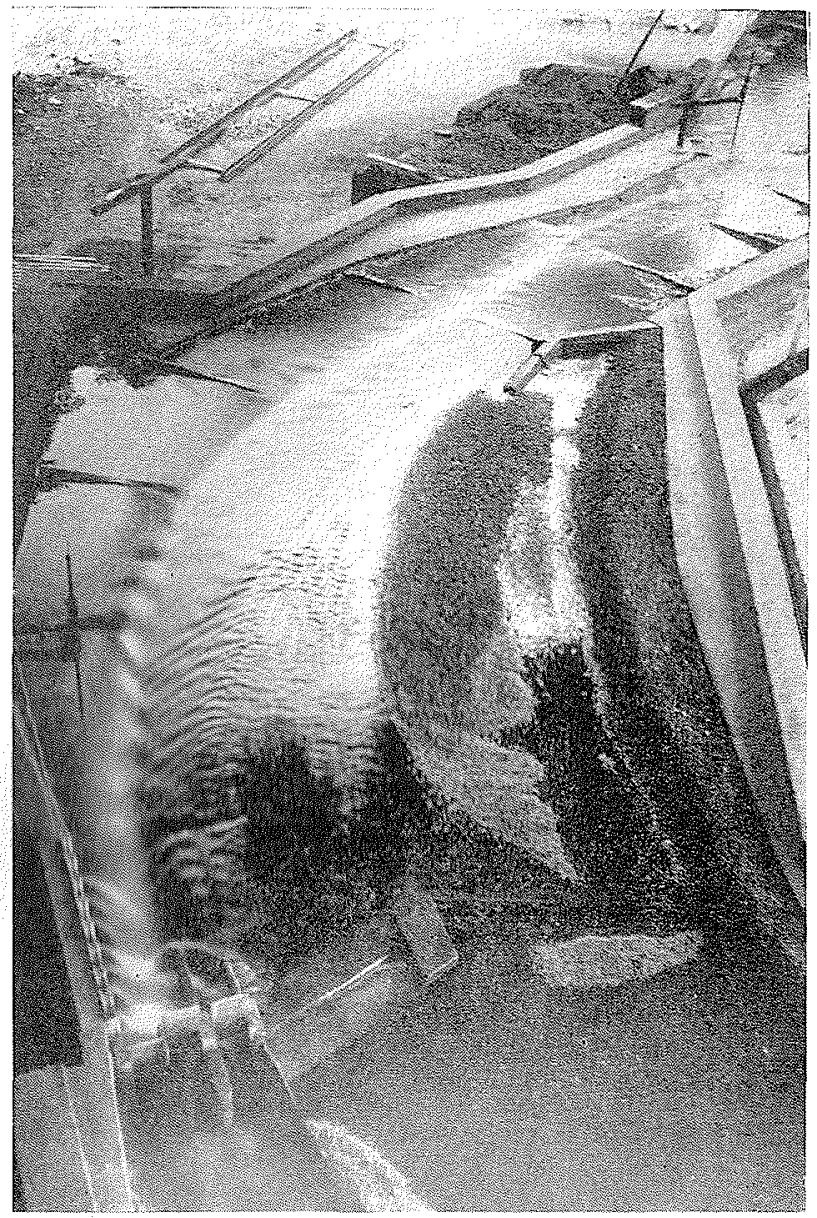

Fro, 7 . Lil amont apres rigularisation.

difications des conditions a l'amont croir lig. (4) et 7 ).

Pour comparer l'efficacité relative des difrerenles modifications apporties aux ouvages, nous avons mesure dans chaque cas le rapport P/C du débit solide $P$ entranl dans la prise au lóhit solide C évacué par les chasses.

Ce rapport était de 0,65 environ lorsqu'on imposial au lit, immédialement avant la prise, un parcours rectiligne el parallèle à la rive droite sur une assez grande longueur; il est devenu praticuenent mal aprés la régularisation du lit à l'amont.

Pour arriver à la création de ces mèmes écoullements transversaux, les hydrauliciens russes utilisent des panneaux de surface orientés convenablement, qui engendrent dans l'écoulement un mouvement hélicoïdal doignant les conches de fond de la prise. L'addition de tels panneaux sur le modele a influé sur lancelioration de la prise au mème degré que la régularisation amont. Cependant, cette solution a été abandonnée en raison du rógime très variable de la Durance.

\section{DISPOSITIFS DE CHASSE}

Il convient de noter à nouveau que le type d'installation étudié correspond au cas où le débit dérivé choisi se trouve inférieur au débit de commencement d'entrainement derant la prise. $\left(\frac{Q_{\prime \prime}}{Q_{0}}=0,6\right)$. On doit donc chercher à disposer dans la mesure du possible des ouvrages de chasses continues. Il faut cependant distinguer de nouveau dans le régime de la rivière un intervalle critique de débit pour lequel le problème de l'évacuation des matériaux sera particulièrement délicat. Il correspond aux débits roisins du dábil de commencement d'entrainement $Q_{0}$.

\section{I. - Intervalle critique de débit}

L'organe principal de chasses est évidemment lit vanne qui doit evacuer à laval tout le charriage de la rivière pour les débits voisins de $Q_{11}$. Nous résumerons done d'abord les observations que nous avons pu fairc à son sujet, pour signaler ensuite les essais relatifs à des dispositifs complémentaires.

\section{a) VaNNE DE CHASSE}

1. Position. - Nous n'insisterons pas sur les avantages quil y a à éloigner de la prise vers le milieu de la rivière la première vanne de chasse utilisée pour régler le niveau à la cote de retenue lorsque le débit de la rivière augmente : on diminue ainsi d'autant les vitesses devant les pertuis.

Plus précisément, il faudrait la placer à l'en. droit ou la bande de charriage se trouve localisće pour le débit Qn par les dispositions prises à l'amont.

Ajoutons enfin que, d'après les resultats de nos cssais, il $y$ a intérét à la placer aussi à lamont que possible des ouvrages de prise et du barrage : on évacue ainsi les matériaux à un endroit où le remous du barrage et la déviation des courants, due à la prise, se font moins sentir et ou, d'autre part, les vitesses sont assez grandes pour (qu'un dispositif de fond puisse contribuer à rassembler les graviers ver's les chasses.

2. Dimensions. - Les deux considérations préédentes, s'ajoutant au point de vue du constructeur, permettent en somme de choisir approximativement la position la plus favorable de la premiere vanne de chasse. Il reste à en fixer les dimensions.

Pour les débits inférieurs à $Q_{0}$, il est nécessaire de maintenir une retenue suffisante pour pouvoir dériver aussi longtemps que possible ic dribit $Q_{n}$ d'équipement. Mais dès que le débil de la rivière est voisin de $Q_{m}$ il convient, pour faci- 
liter l'évacuation des matériaux, de réaliser un foulement libre à travers une bouchure suffisalmment étroite pour que la vitesse d'approche des filets liquides qui l'alinente soit assez nettement supérieure à la vitesse de l'eau au droil de la prise. La cote aval étant suffisamment abaissée et celle du radier étant choisie (pour la Saulce, elle etait à environ $1 \mathrm{~m}$ en dessous du fond naturel), on connait le niveau de l'eau qui existerait devant la vanne el sans les ouvages pour le débit $\mathrm{Q}_{0}$; il est alors possible d'en déduire la largeur de louverture nćcessaire pour ivacuer en regime crilique le débil Q. - Q .. En fait, vu l'insuffisance actuelle de calculs alpplicués à des phénomènes si complexes, toutes ces considéralions ne permettront de déterminer qu'un ordre de grandeur des dimensions à préconiser.

\section{b) OUVRAgES SECONDAIRES}

Ils ont pour but de concentrer les matériaux solides dans la zone d'aclion de la vanne de chasse en augmentant autant que possible la force tractrice. On cherchera en somme à accroìtre l'écoulement transversal dù à la correction. amont et s'opposant à celui dû à la présence de la dérivation. Ceci conduit à différentes dispositions favorables au dégravement de la prise.

1. Comme l’ont déjà montré de nombreux essais, notamment ceux de M. Ic professeur MrexerPETer pour la prise de Lavey, il y a intérêt à ménager, entre la prise el la première vanne de chasse, une sorte de « canal de prise» limité par un mur guideau. On observe alors au voisinage de l'extrémite amont de celui-ci que les

\section{LEGENDE}

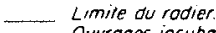

Ouvrages insubmersibles.

oifferentes dimensions essoyes des murc de séparolion. vonnes droites.

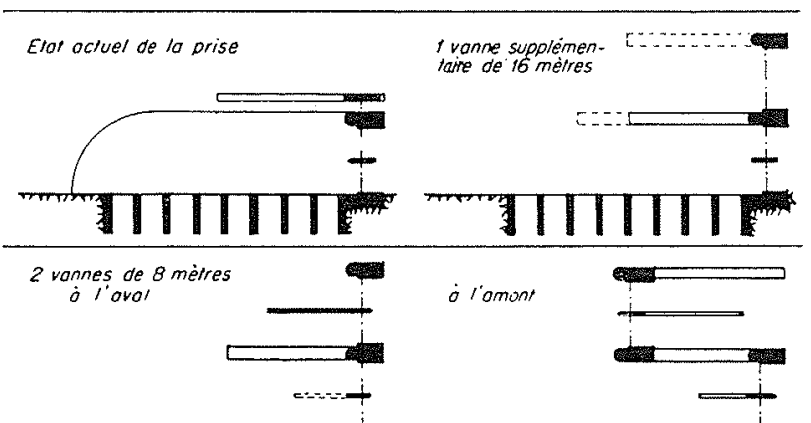

M TIIIIIIF

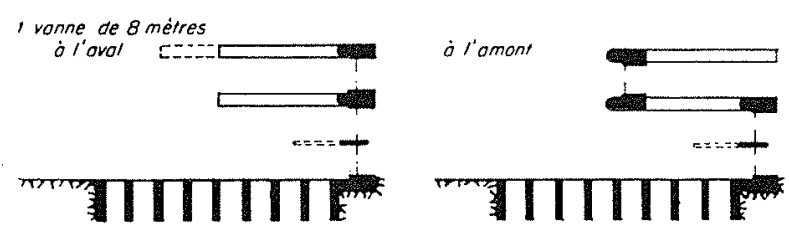

Fra. 8. - Essais de modifications des vannes de chasse. courants de fond sont allirés de ce canal vers les chasses : à cet endroit, les filets liquides de surface et de fond arrivent a faire entre cux un angle supérieur à $90 "$.

Il convient cependant, pour plus de sécurite, de eonserver à liaval du canal de prise une vanne permeltant d'efrectuer des chasses discontinues de son radier en fermant la prise.

(Pour l'ensemble des modifications faites au cours des essais décrits ci-dessus, voir fig. 8.)

2. Nous avons cherché à déterminer les formes a donner au seuil de cet avant canal situé entre te mur guideau et la prise, de facon a accentuer encore la déviation des filets de fond vers les chasses. Nous avons entrepris à cet eflet, dans un canal vilré de $1 \mathrm{~m}$ de largeur, une étude

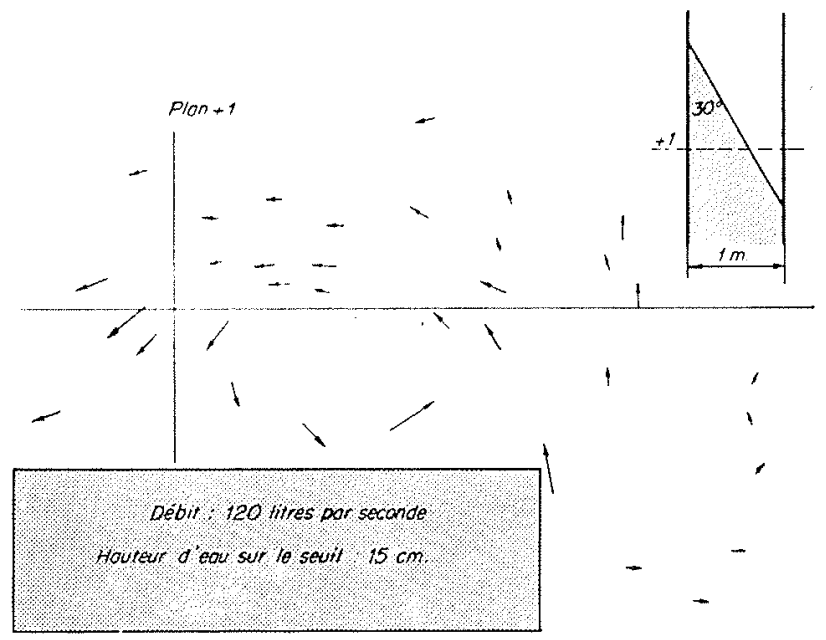

Fic. 9, - Projection des vitesses sur un plan normal.

systématique de laction de différents seuils sur la trajectoire des filets liquides au voisinage du fond.

Nous avons pu ainsi établir le champ des vitesses au voisinage du seuil. Les vitesses des particules dans un plan donné, perpendiculaire à l'axe du canal, ont été projetées sur ce plan, de façon à en analyser les composantes transversales (cf. fig. 9). Pour chaque inclinaison de seuil, cette opération était répétée pour plusieurs plans. L'examen des résultats ainsi obtenus montre que le seuil crée non seulement une circulation transversale, mais engendre également un tourbillon de décollement.

Les figures 10, 11, 12 permettent de suivre, d'autre part, la déformation des trajectoires selon leur arrivée par rapport au seuil. La figure 13 donne enfin leur aspect expérimental.

3. Ceci a conduit à étudier la disposition suivante : on placait sur le fond du canal un premier seuil noccupant pas toute la largeur; il 

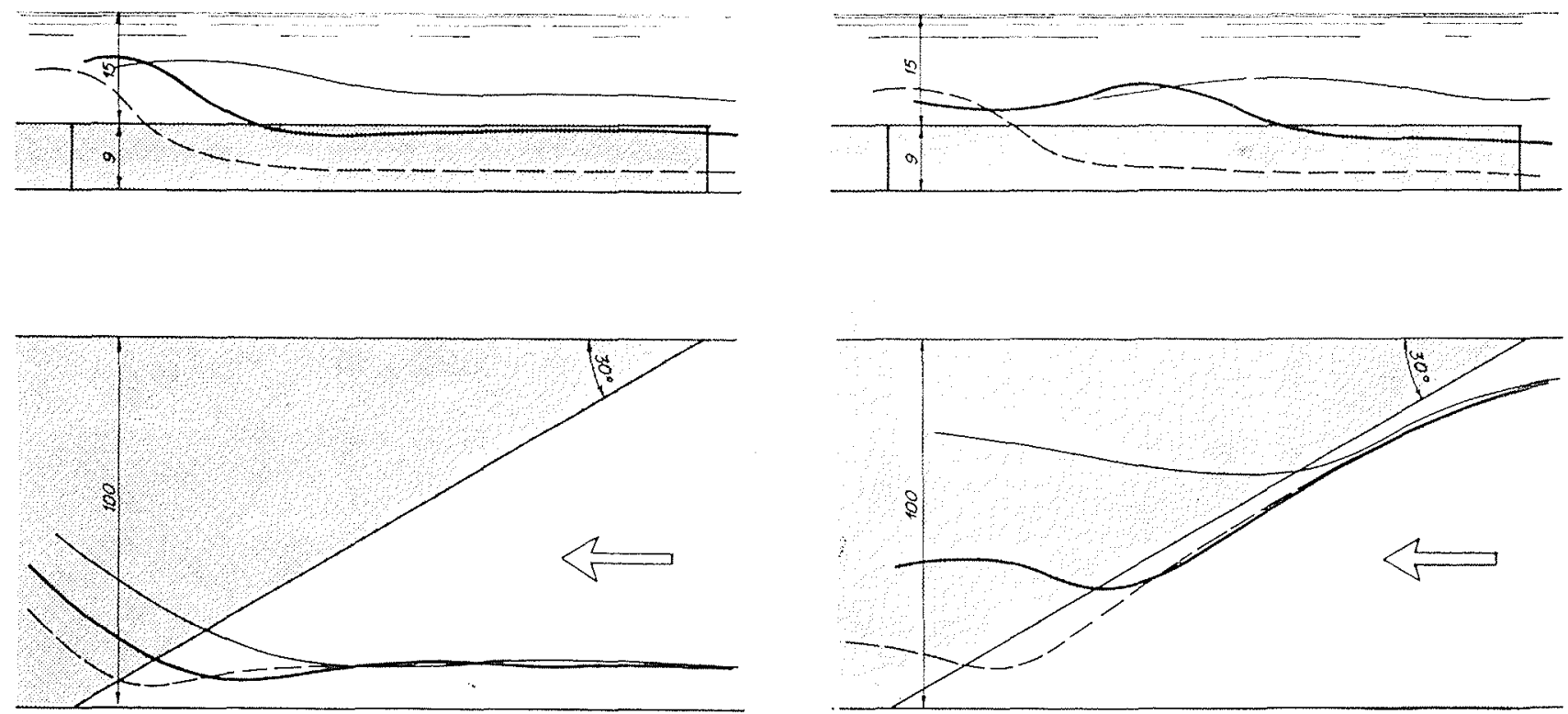

F'ri. 10. - Aspect des lignes de courant athequant le seuil à l'aval à différentes hauteurs.

Fia. 12. - Aspect des lignes de conrant attaquant le seuil à l'amont à differentes hauteurs.

laires de $8 \mathrm{~m}$ de largeur, te rapport $\frac{P}{C}$ passe de 1,5 à 0,65 avec le double seuil. Cependant, comme après la régularisation l'on ne constatait pratiquement plus d'entrie des matériaux dans la prise, il n’apparut pas nécessaire de pousser plus loin l'étude.

\section{II. - Débits supérieurs à ceux de l'intervalle critique}

Il s'agil alors en principe de réaliser artifieiel-

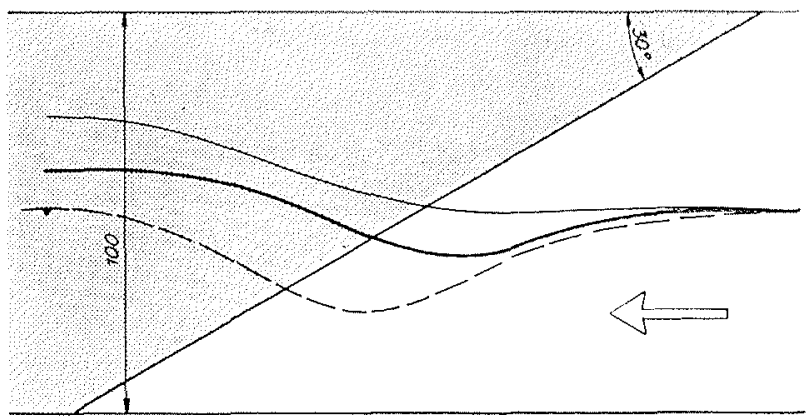

Fic. 11. - Aspect des lignes de courant attaquant le seuit au milien a differentes hauteurs.

était suivi d'un second seuil de même section, un peu plus long, en contact par son extrémité amont avec la paroi du canal.

En espaçant convenablement les deux seuils, le tourbillon engendré par le premier se développait à l'amont du second el l'on observait dans la rainure ainsi ménagée un courant traversier hélicoïdal favorable à l'entrainement des matériaux (cf. fig. 14).

Celte disposition, essayée sur le modèle, a donné satisfaction, quoique le premier seuil soit partiellement engravé à l'amont. Sans la régularisation amont et avec deux vannes supplémen.
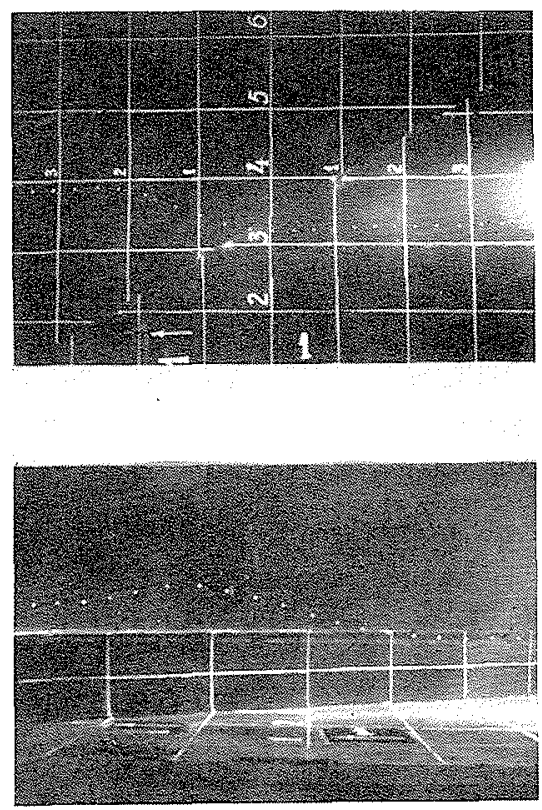

Fia. 13. - Vue verticale et latemale d'une trajectoire expérimentale. 
el la largeur de charriage varient selon le dóbit daprès les mèmes lois qu'un lit naturel au droit des ouvages. On devrait done prévoir dans linsstallation des bouchures rejoignant le déversoir de

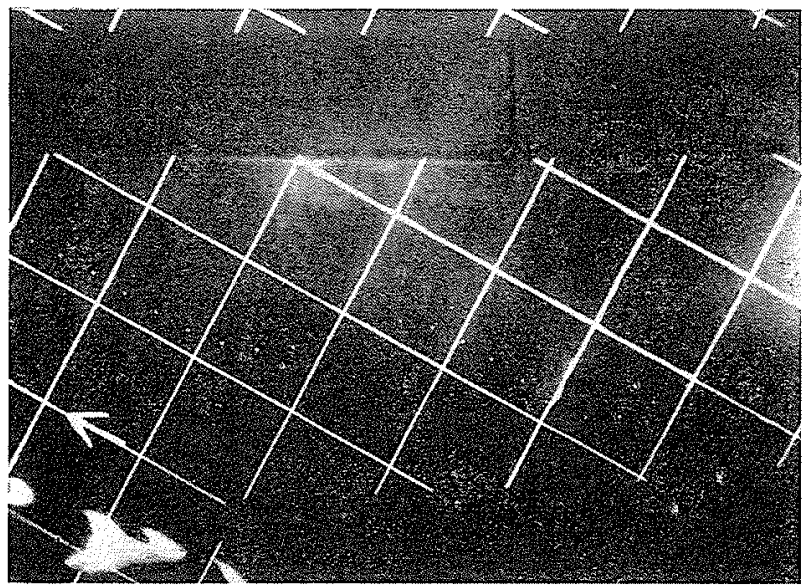

Fio. 14. -- Mise en évidenec du coulant hélicoïdal entre deux souils.

(Direction du comrent suibant la fieche)

sécurité d'une manière continuc et selon les mêmes principes que pour la ranne de lintervalle critique. Fn lait, pour des questions d'économic el de simplicité par rapport aux ouvrages existants, nous avons seulement ajouté sur le modele deux nouvelles vannes de $8 \mathrm{~m}$ de largeur, contiguës à la vanne que nous avions dejà prévue pour les débits voisins de $\mathrm{Q}_{0}$.

Nous avons constaté cependant que cette disposition préscnte des inconvénients qui semblent d'ailleurs pouvoir exister, même si les ouvrages de chasse etaient mieux réalisés : au cours des crues importantes, le lit mineur s'élargit en direction du barrage et, lorsque le débit revient à une valeur plus faible, le creusement du chenai à sa nouvelle section se fail tres lentement ef pas toujours d'une manière favorable.

Pour obtenir une solution réellement satisfaisante, il s'agissait done de prolonger le lit amonl rectifié par un chenal auto-stable, malgré l'effet du remous el le déplacement de la zone de charriage vers lintérieur du coude avec l'angmentation du débit de la rivière. Dans ce but, on a disposé, sur l'intérieur de la courbe, à l'anont du barrage, une séric d'ouvrages, perméables à la fois all débit solide et au débil liquide et représentés sur le modele par des lignes de pieux perpendiculaires au courant. On constate alors, au cours de la décrue, un dépòt de matériaux dans la zone d'élargissement des erues. Le lit se creuse ainsi à nourcau assez rapidement vers la vanne de chasse correspondant ì l'intervalle critique de débit (ef. lig. 15).

Dans le cas éludié de la prise de la Saulee, cette disposition presentait d'autre part lintérèt de concentrer le débit solide vers le chenal évacuateur en supprimant pratiquement le débit solide sur le barrage; on limitait ainsi à la fois l'exhaussement du lit intermiltent cré par déversement sur le barrage et l'érosion du chenal a la suite d'une insuffisance, ou mème, pour les débits voisins de $Q_{0}$, d'une alusence d'alimentation en matériaux.

Il reste évidemment à transposer de tels ouvrages à la réalité. Cela peut présenter certaines diflicultes, surtoul a cause du mancque dexpiriences que l'on possede à leur sujet en France.

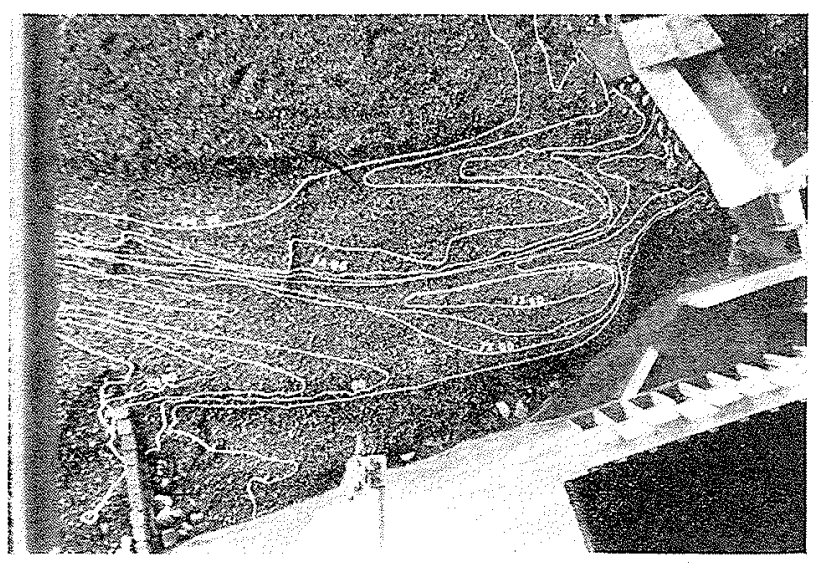

Fir. 15. - Relief du fond devant la prise après modifications.

Comparćs à des défenses par épis, ils paraissent pourtant plus économiques ef mieux adaptés aux conditions naturelles.

\section{CONCLUSION}

L'étude sur modèle a permis de proposer une solution satisfaisante pout lutter contre l'engravement de la prise de la Saulce. Le prix des travaux à exécuter parait sans doute élevé quand on considere d'une part qu'il s'agit de la modification d'une prise existante ef, d'autre part, que la construction probable du barrage de SerrePonçon posera le problème du transport solide 
a la Saulce d'une facon toute difrérente, fonction du mode d'exploitation, non précisé encore, du réservoir. Cependant, dans le cas d'une prise en projet, il est possible, par des essais sur modele, de déterminer, sans augmentation prohibitive du coûl de construction, des formes d'ouvages permettant de se prémunir au maximum contre les engravements. La question des modifications locales de profils en long el des seclions transversales du lit serait plus facile à résoudre si lon connaissait mieux les lois qui les relient au régime global des cours d'eau. Ia détermination de ces lois demande une etude statistique dans la nature, sur des données qui sont actuellement trop peu nombreuses, mais dont l'inter- prétation sera facilite par des hypotheses à priori. Celles que nous avons mises au début de cette note peuvent servir de base de départ et si elles s'avèrent fausses, peut-être verra-ton apparaitre au cours du travail de nouvelles corrélations plus fructueuses.

\section{Commextahe de Préshent}

M. le Président remercie M. LABAyE d'avoin fait un exposé aussi interessant sur les résultats de ses essais relatifs au degravement de la prise de la Saulce sur la Durance. Les nombreuses observations qu'il a pu faire a lappui de ses hypotheses et les déductions qui en découlent apportent une documentation supplementaire a l'expérience des spécialistes.

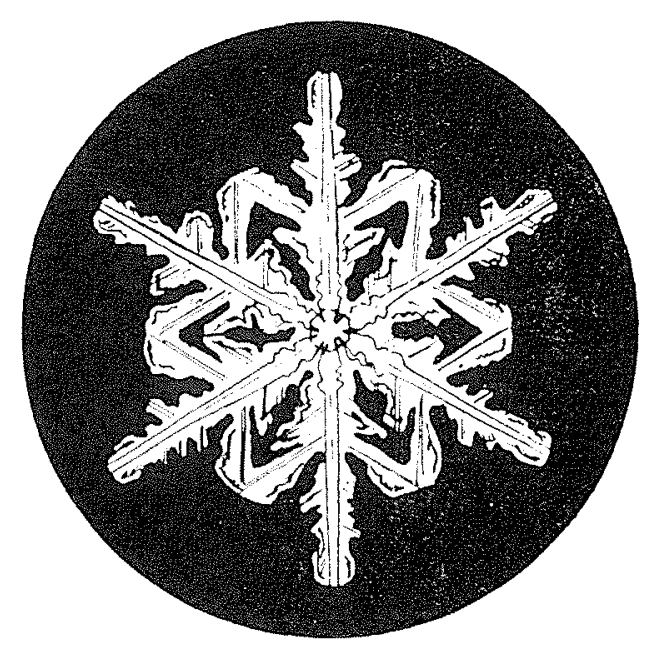

